\title{
Adição ao trabalho e relação com fatores de risco sociodemográficos, laborais e psicossociais
}

\author{
Mary Sandra Carlotto 1 - Pontifícia Universidade Católica do Rio Grande do Sul, Porto Alegre, Brasil
}

\begin{abstract}
Resumo
O estudo buscou Identificar a prevalência e os fatores de risco sociodemográficos, laborais e psicossociais da adição ao trabalho em 471 trabalhadores de Porto Alegre e região metropolitana. Como instrumento foi utilizado o "Dutch Work Addiction Scale" (DUWAS), versão reduzida. A escala avalia a adição ao trabalho em suas duas dimensões, o Trabalho Compulsivo e Trabalho Excessivo. Os resultados revelam que, dentre as variáveis sociodemográficas, somente a variável sexo assinalou diferença significativa, tendo as mulheres apresentado índices mais elevados em Trabalho Excessivo. Quanto às variáveis laborais, verificou-se associação positiva entre Trabalho Excessivo e carga horária contratual, e Trabalho Excessivo e carga horária efetivamente realizada. Houve associação negativa entre Trabalho Excessivo e percepção de estar saudável. O Trabalho Compulsivo se associou negativamente ao tempo de exercício profissional e de trabalho na empresa atual, bem como à percepção de estar saudável e à satisfação com a vida em geral. Palavras-chave: Adição ao trabalho, Trabalho compulsivo, Trabalho excessivo, Fatores de risco.
\end{abstract}

Workaholism and relationship with sociodemographic, work and psychosocial risk factors

\begin{abstract}
The goal of this study is to identify sociodemographic, laboral and psychosocial risk factors of workaholism in 471 workers in Porto Alegre and metropolitan area. The instrument used was the reduced version of the Dutch Work Addiction Scale (DUWAS). The scale assesses workaholism in its two dimensions, Excessive Work and Compulsive Work. The results showed that from the sociodemographic variables, only the gender variable showed a significant difference, where women had higher rates of excessive work. As for the labor variables, Excessive Work had a positive association with contractual working hours and with working hours that were effectively carried out; and a negative association with the perception of being healthy. Compulsive Work was negatively correlated to the time of professional practice and of work in the current company, to the perception of being healthy, and to satisfaction with life in general. Keywords: Workaholism, Excessive work, Compulsive work, Risk factors.
\end{abstract}

A adição ao trabalho ou workaholism, expressão americana que tem origem na palavra alcoholic (alcoólatra), foi utilizada para designar uma pessoa viciada em trabalho (Salanova, Del Libano, Llorens, Schaufeli \& Fidalgo, 2008). De acordo com Killinger (1991), a adição ao trabalho é um processo no qual, gradualmente, a pessoa vai perdendo sua estabilidade emocional e o controle sobre o trabalho em uma tentativa compulsiva de obter êxito constante. Cherrington (1980) complementa destacando o caráter irracional do trabalho excessivo, pois a pessoa se torna incapaz de encontrar outras formas de ocupação além da sua atividade laboral.

As pessoas podem trabalhar muitas horas devido a problemas financeiros, pobre relacionamento conjugal ou familiar, forte intenção de ascender profissionalmente, sem que sejam adictos ao trabalho. O que diferencia os adictos é o desejo obsessivo com evidente falta de controle sobre o trabalho e as horas dedicadas ao mesmo (Schaufeli, Taris \& Bakker, 2008). É importante destacar que não se trata de trabalhar muito, mas de trabalhar além do solicitado (Scott, Moore \& Miceli, 1997; Serva \& Ferreira, 2006),

\footnotetext{
${ }^{1}$ Endereço para correspondência:

Av. Mauá, 645, apto 504 - Centro - São Leopoldo/RS. CEP: 93110-320

E-mail: mscarlotto@pesquisador.cnpq.br
}

estendendo parte das atividades para os contextos extralaborais (Salanova \& cols., 2008). Os trabalhadores dependentes do trabalho fazem projetos simples ficarem mais complicados do que o necessário. Trabalham mais tempo e mais arduamente do que os outros, não porque os seus empregos os obrigam a fazê-lo, mas porque eles tendem a criar, para si mesmos, altas exigências com seu trabalho (Schaufeli, Taris \& Bakker, 2008).

Nas diversas definições encontradas, sempre, estão contempladas a conduta dedicada, seu caráter compulsivo e a baixa capacidade de usufruir do trabalho e dos resultados obtidos com o mesmo (Moreno-Jiménez, Gálvez-Herrer, Garrosa-Hernández \& Rodríguez Carvajal, 2005). De acordo com os autores, ocorre a presença de envolvimento progressivo, excessivo e desadaptativo frente ao trabalho, com clara perda de limites, com ações voltadas mais para uma necessidade pessoal do que organizacional.

Ainda que o termo workabolic, seja bem conhecido na linguagem cotidiana, surpreende o fato de ainda se saber tão pouco, do ponto de vista científico, sobre as suas causas e consequências, sendo que nem mesmo sua conceituação possui um acordo (Salanova \& cols., 2008; Taris \& Schaufeli, 2007).

Vários estudos têm sido desenvolvidos (Bakker, Demerouti \& Burke, 2009; Burke, 2000a, 2000b, 2003, 2004, 2008; Del Líbano, Renedo, Lorens 
\& Salanova, 2005; Del Líbano, Llorens, Salanova \& Schaufeli, 2010; Dudek, 2008; Porter, 1996; Serva \& Ferreira, 2006; Schaufeli, Taris \& Bakker, 2008; Shimazu \& Schaufeli, 2009; Snir \& Zohar, 2008; Spence \& Robbins, 1992; Taris, Schaufeli \& Verhoeven, 2005), no entanto o conhecimento de sua natureza, fatores antecedentes e consequências seguem mantendo zonas de incerteza e desconhecimento (Moreno-Jiménez, Gálvez-Herrer, Garrosa-Hernández \& Rodríguez-Carvajal, 2005).

Conforme Thomas, Sorensen e Feldman (2006), a imprensa popular tem oferecido mais informações do que a científica. No Brasil, o que se verifica, hoje, é uma grande quantidade de informações com definições gerais e orientações voltadas para a autoajuda, geralmente, executadas por sites comerciais. Para o público em geral, o conceito é sinônimo de muitas horas de trabalho extremamente difíceis e não leva em consideração a adição do trabalhador em relação ao trabalho (Burke, 2008; Shimazu \& Schaufeli, 2009). A adição ao trabalho tem mostrado crescimento no atual contexto laboral (Hewlett \& Luce, 2006). Algumas culturas corporativas estimulam e privilegiam as condutas que ultrapassam o contrato funcional de trabalho, os horários oficiais estabelecidos, sem preocupar-se com os efeitos dessa conduta no âmbito pessoal e familiar (Moreno-Jiménez, Gálvez-Herrer, Garrosa-Hernández \& Rodríguez-Carvajal, 2005). Estudo realizado com 470 trabalhadores espanhóis por Salanova, Llorens, Cifre e Martínez (2006) identificou que $79 \%$ dos sujeitos apresentavam indicadores de adição ao trabalho.

Assim, tendo em vista a relevância do tema para possíveis intervenções no campo da Saúde Mental do Trabalhador, este estudo observacional analítico transversal buscou identificar os fatores de risco sociodemográficos, laborais e psicossociais da adição ao trabalho em uma amostra multifuncional de trabalhadores de Porto Alegre e região metropolitana.

\section{Método}

\section{Participantes}

A escala foi aplicada em 471 trabalhadores, independente de sexo e escolaridade, que exercem suas atividades em organizações de trabalho localizadas em Porto Alegre e região metropolitana, RS, Brasil, 2009. Como critério de inclusão os participantes deveriam desenvolver seu trabalho em uma carga horária semanal superior a 40 horas.

\section{Instrumentos}

Para o levantamento das variáveis sociodemográficas (sexo, idade, situação conjugal, escolaridade, formação, remuneração), laborais (função, tempo de exercício profissional, tempo de exercício profissional na empresa atual, carga horária semanal e contratual, quantidade de horas efetivamente trabalhadas, vínculo empregatício, trabalhar em mais de uma instituição), psicossociais (percepção de saúde avaliada considerando um escala tipo likert de cinco pontos, variando de $1=$ ruim a $5=$ excelente; satisfação com a vida avaliada considerando um escala tipo likert de cinco pontos, variando de $1=$ nada insatisfeito a $5=$ muito satisfeito; número de horas dedicadas ao lazer) foi elaborado um questionário auto-aplicável baseado no referencial teórico sobre o tema visando a responder os objetivos do estudo.

A adição ao trabalho foi avaliada utilizando a versão reduzida da "Dutch Work Addiction Scale" (DUWAS), de Schaufeli, Taris e Bakker (2006). A escala avalia a adição ao trabalho em suas duas principais dimensões, o Trabalho Compulsivo (TC) e o Trabalho Excessivo (TE). No total, constitui-se de 10 itens avaliados por uma escala tipo likert, variando de 0 (nunca) a 3 (todos os dias). A escala já se encontra validada no Japão (Schaufeli, Shimazu \& Taris, 2009) e na Espanha (Del Libano \& cols., 2010), e encontra-se disponível no site de Wilmar Schaufeli (http://www.schaufeli.com).

Adaptação da escala (tradução para o português do Brasil) e estudo prévio das propriedades psicométricas (validade de conteúdo, validade de construto, confiabilidade), realizado por Carlotto e Del Líbano (2010), identificaram que o modelo teórico se ajusta satisfatoriamente aos dados, confirmando sua estrutura bifatorial. Os dois fatores apresentaram alfa de Cronbach superior a 0,70. Resultados mostram que a escala reduzida oferece validade fatorial e consistência interna adequada para avaliar a adição ao trabalho em profissionais brasileiros.

\section{Procedimentos}

Foram realizados os procedimentos éticos conforme Resolução 196 do Conselho Nacional de Saúde (Ministério da Saúde, 1997), no que diz respeito à pesquisa com seres humanos. O estudo possui aprovação pelo Comitê de Ética em Pesquisa da instituição de afiliação da autora. Foi esclarecido aos sujeitos e diretores e /ou gerentes das instituições de trabalho mostrando tratar-se de uma pesquisa sem quaisquer efeitos avaliativos individuais $\mathrm{e} / \mathrm{ou}$ institucionais; sendo as respostas e os dados referentes aos resultados anônimos e confidenciais. 
A coleta de dados foi realizada no período de março a outubro de 2009 por três estudantes de psicologia, bolsistas de iniciação científica do Laboratório de Ensino e Pesquisa em Psicologia (LAEPPSI-ULBRA/Canoas), vinculados ao projeto. Estes foram treinados a fim de resguardar os procedimentos de padronização na aplicação. Após autorização, os instrumentos foram aplicados em grupo em alunos/trabalhadores em salas de aulas de uma universidade particular e recolhidos logo após o preenchimento. Em uma segunda etapa, utilizou-se a técnica de recrutamento em cadeia (bola-de-neve) (Biernacki \& Waldorf, 1981), em que os primeiros respondentes indicam outros, os quais, por sua vez, indicam outros e assim sucessivamente. Após indicação, alunos do LAEPPSI entraram em contato para convidar à participação no estudo. Os instrumentos foram entregues no intervalo de trabalho dos participantes e recolhidos após o seu preenchimento.

O banco de dados foi analisado por meio do programa SPSS - Statistical Package for Social Sciences, versão 13.0, sendo realizada análise descritiva de caráter exploratório no intuito de avaliar a distribuição dos itens, casos omissos e identificação de extremos. A análise descritiva foi realizada para caracterizar a população estudada, apresentar os índices médios de cada dimensão da variável desfecho adição ao trabalho e determinar sua prevalência. A análise da prevalência da seguiu os procedimentos dos pontos de referência da escala tipo Likert na qual os sujeitos atribuem os valores para os ítens da escala baseados na freqüência de sintomas (Shirom,1989). Este procedimento é indicado para utilização em países que ainda não possuem pontos de corte validados.

Posteriormente, utilizou-se a correlação de Pearson para medir o grau de relação linear entre adição ao trabalho e variáveis preditoras qualitativas ordinais. Também, foram utilizados o teste $t$ de Student a fim de comparar duas médias dos escores das variáveis e a ANOVA para comparar três ou mais grupos de escores médios, complementada pelo teste de Tukey (post hoc), sendo adotado o nível de significância de 5\%.

\section{Resultados}

Os trabalhadores que constituem a população em estudo são, em sua maioria, mulheres $(56,2 \%)$, com idade média de 34,7 anos (DP $\pm 11,8$ ), solteiros/separados $(52,2 \%)$, com companheiro fixo $(75,7 \%)$, com filhos $(54,6 \%)$ e possuem remuneração de até 3 salários mínimos $(55,9 \%)$. Em relação à escolaridade, $12,2 \%$ possuem ensino fundamental, $32,6 \%$ ensino médio, $26,9 \%$ superior incompleto, $21,9 \%$ superior e $6,5 \%$ formação técnica. A maioria dos trabalhadores possui vínculo empregatício $(67,6 \%)$ e trabalham em um único local $(83,5 \%)$. Possuem 12,9 anos (DP $\pm 10,8)$ de trabalho, 6 anos de trabalho no local (DP $\pm 4,98)$, realizam, em média, 44 horas semanais de trabalho contratual $(\mathrm{DP} \pm 6,2)$ e 49 horas de trabalho efetivo (DP $\pm 11,8)$.

Resultados revelam que mais de um terço apresentam alto nível de trabalho excessivo e 14,6\% de trabalho compulsivo (Tabela 1).

Tabela 1 - Prevalência das dimensões da adição ao trabalho $(\mathrm{n}=471)$

\begin{tabular}{|c|c|c|c|c|}
\hline \multirow[t]{2}{*}{ Dimensões } & \multicolumn{2}{|c|}{$\begin{array}{l}\text { Baixo nível } \\
\mathrm{M}<3\end{array}$} & \multicolumn{2}{|c|}{$\begin{array}{l}\text { Alto nível } \\
\mathrm{M} \geq 3\end{array}$} \\
\hline & $\mathrm{n}$ & $\%$ & $\mathrm{n}$ & $\%$ \\
\hline Trabalho Excessivo & 306 & 65,0 & 165 & 35,0 \\
\hline Trabalho Compulsivo & 402 & 85,4 & 69 & 14,6 \\
\hline
\end{tabular}

Com relação às variáveis qualitativas, somente ocorreu diferença significativa entre o grupo de homens e de mulheres. Trabalhadoras apresentaram médias mais elevadas em Trabalho Excessivo (Tabela 2).

Resultados obtidos na matriz de correlação evidenciam associação positiva entre Trabalho
Excessivo e carga horária contratual e a efetivamente realizada e negativa com a percepção de estar saudável. O Trabalho Compulsivo associou-se negativamente ao tempo de exercício profissional e local, assim como a percepção de estar saudável e satisfação com a vida em geral (Tabela 2). 
Tabela 2 - Relação entre dimensões de adição ao trabalho e variáveis qualitativas

\begin{tabular}{|c|c|c|c|c|c|c|c|}
\hline \multirow{2}{*}{$\begin{array}{l}\text { Dimensões } \\
\text { Variáveis }\end{array}$} & \multirow[b]{2}{*}{$\mathrm{n}$} & \multicolumn{3}{|c|}{ TE } & \multicolumn{3}{|c|}{$\mathrm{TC}$} \\
\hline & & $\mathrm{M}$ & $\mathrm{DP}$ & $\mathrm{p}$ & $\mathrm{M}$ & $\mathrm{DP}$ & $\mathrm{p}$ \\
\hline \multicolumn{8}{|l|}{ Sexo } \\
\hline Masculino & 206 & 1,56 & 0,64 & $0,04 *$ & 2,00 & 0,67 & 0,31 \\
\hline Feminino & 264 & 1,68 & 0,68 & & 1,93 & 0,62 & \\
\hline \multicolumn{8}{|l|}{ Rel. Pessoais } \\
\hline Com companheiro & 330 & 1,65 & 0,69 & 0,82 & 1,14 & 0,67 & 0,13 \\
\hline Sem companheiro & 106 & 1,63 & 0,62 & & 1,25 & 0,65 & \\
\hline \multicolumn{8}{|l|}{ Filhos } \\
\hline Não & 213 & 1,63 & 0,67 & 0,88 & 1,89 & 0,64 & 0,68 \\
\hline Sim & 256 & 1,64 & 0,66 & & 2,03 & 0,70 & \\
\hline \multicolumn{8}{|l|}{ Escolaridade } \\
\hline Fundamental & 49 & 1,66 & 0,69 & 0,95 & 1,19 & 0,79 & 0,91 \\
\hline Médio & 131 & 1,61 & 0,62 & & 1,19 & 0,63 & \\
\hline Superior incompleto & 108 & 1,63 & 0,68 & & 1,16 & 0,66 & \\
\hline Superior completo & 88 & 1,57 & 0,67 & & 1,11 & 0,69 & \\
\hline Técnico & 26 & 1,63 & 0,69 & & 1,12 & 0,75 & \\
\hline \multicolumn{8}{|l|}{ Reside } \\
\hline Sozinho & 49 & 1,70 & 0,65 & 0,75 & 1,16 & 0,69 & 0,64 \\
\hline Companheiro e/ou filhos & 296 & 1,63 & 0,68 & & 1,13 & 0,66 & \\
\hline Pais & 119 & 1,63 & 0,65 & & 1,20 & 0,68 & \\
\hline \multicolumn{8}{|l|}{ Remuneração } \\
\hline Até 3 SM & 246 & 1,59 & 0,67 & 0,50 & 1,16 & 0,67 & 0,83 \\
\hline De 3 a 6 SM & 119 & 1,65 & 0,63 & & 1,11 & 0,65 & \\
\hline Mais de $6 \mathrm{SM}$ & 75 & 1,67 & 0,70 & & 1,14 & 0,77 & \\
\hline
\end{tabular}

Nota: *Diferença significativa ao nível de $5 \%$.

$\mathrm{TE}=$ Trabalho Excessivo; $\mathrm{TC}=$ Trabalho Compulsivo 
Tabela 3 - Matriz de correlação entre dimensões da adição ao trabalho e variáveis quantitativas

\begin{tabular}{lcc}
\hline Variáveis & TE & TC \\
\hline Idade & $-0,010$ & $-0,045$ \\
Tempo de exercício profissional & $-0,020$ & $-0,095^{*}$ \\
Tempo de exercício profissional na empresa atual & $-0,034$ & $-0,108^{*}$ \\
Carga horária semanal contratual & $0,148^{* *}$ & $0,100^{*}$ \\
Carga horária efetivamente trabalhada na semana & $0,206^{* *}$ & $0,108^{*}$ \\
Tempo de deslocamento casa/trabalho e trabalho/casa & $-0,023$ & $-0,039$ \\
Percepção de saúde & $-0,181^{* *}$ & $-0,129 * *$ \\
Dias (últimos 12 meses) não trabalhados devido a doença & 0,035 & $-0,040$ \\
Satisfação com a vida & $-0,087$ & $-0,132^{* *}$ \\
Horas de lazer & 0,078 & 0,050
\end{tabular}

Nota: * Correlação significativa ao nível de 5\%

** Correlação significativa ao nível de 1\%

$\mathrm{TE}=$ Trabalho Excessivo; $\mathrm{TC}=$ Trabalho Compulsivo

\section{Discussão}

O objetivo do estudo foi identificar a prevalência e os fatores de risco sociodemográficos, laborais e psicossociais da adição ao trabalho. No que diz respeito à prevalência verifica-se um percentual elevado de alto nível de trabalho excessivo, mais de um terço dos investigados, e preocupante de trabalho compulsivo, uma vez que 69 trabalhadores $(14,6 \%)$ já não possuem controle sobre sua atividade de trabalho.

Quanto ao perfil de risco, resultados evidenciam um perfil de TE constituído de mulheres, profissionais com carga horária contratual e efetiva de trabalho mais elevada e que se percebem menos saudáveis. No perfil de TC, identificam-se menor tempo de exercício profissional e local, maior carga horária contratual e efetiva realizada, percepção de não estar saudável e menor satisfação com a vida em geral.

Os resultados encontrados concernentes às mulheres podem ser entendidos pela questão, já bastante consistente na literatura, sobre a dupla jornada exercida por mulheres profissionais. A progressiva incorporação da mulher ao mercado de trabalho faz com que a mesma tenha que se dividir entre a atividade profissional e os trabalhos domésticos (Apple, 1995). Mesmo que compartilhe tarefas com seu companheiro, ela, ainda, é responsável pela maior parte delas (Codo, 1999). Além de algumas condições biológicas e sociais que intervêm de maneira importante nas variações de saúde de homens e mulheres, como a função reprodutiva, o trabalho doméstico e a atividade produtiva, existem fatores relacionados à valorização social de gênero de algumas profissões que se constituem em fatores de risco de doenças ocupacionais (Borrell \& Artazcoz, 2008; Miranda \& Lobato, 2009).

No que diz respeito às variáveis laborais, verifica-se que quanto maior a carga horária contratual e a efetivamente realizada mais elevados são o TE e o TC. Resultado semelhante foi identificado em estudo desenvolvido por Schaufeli, Taris e Bakker (2008), com trabalhadores holandeses. Os autores destacam a importância e relevância desse resultado para caracterizar a adição ao trabalho, uma vez que esses trabalhadores costumam sobrecarregar-se e extrapolar os horários oficiais de trabalho. Segundo Porter (2004), há duas motivações para trabalhar muitas horas, ou a pessoa gosta de sua atividade, ou é motivada pela competitividade e manutenção de seu trabalho. Tucker e Rutherford (2005) complementam referindo que uma das razões é porque o trabalho lhe proporcina crescimento, prazer e recompensas. A outra seria pela insegurança, temor de sanções negativas por parte da chefia. Um estudo de Burke com trabalhadores canadenses (2000b) identificou que workabolics não executam seu trabalho na busca de aumentos salariais, satisfação na carreira ou qualquer outra recompensa pelos seus esforços.

Também pode se pensar em um processo fruto da denominada Indústria Cultural, na qual, segundo Adorno e Horkheimer (1985), o trabalhador é considerado um instrumento de trabalho e de consumo. Sua manipulação e ideologização fazem com que até mesmo o seu lazer se torne uma extensão do 
trabalho. Ao proporcionar constantemente novas necessidades provenientes do sistema vigente, o trabalhador passa a sentir-se frequentemente insatisfeito e desenvolve a sensação de ter que trabalhar mais para atingir o sempre novo patamar de consumo.

O TC associou-se negativamente ao tempo de exercício profissional e local. Quanto menor o tempo de trabalho geral e no local atual maior é o componente compulsivo no trabalho. Pode se pensar na relação desse resultado, dentro do atual contexto competitivo, por estarem há pouco tempo no mercado de trabalho e na organização, assim, os trabalhadores acabam por sobrecarregar-se de atividades, então, desenvolvendo paralelamente o TC. Segundo Serva e Ferreira (2006), o trabalhador para atender a esta realidade altamente competitiva de um mercado turbulento e descontínuo, passa a comporta-se de acordo com perfil cada vez exigente. As organizações cada vez mais estão buscando um trabalhador mais absorvido, altamente empenhado, comprometido e dedicado. Uma dedicação integral e uma vida quase exclusivamente voltada para a empresa parecem ser a regra de sobrevivência nas organizações modernas e o caminho único de sucesso na carreira.

Quanto menos saudável percebe-se o trabalhador maior o TE e TC. Esse resultado é consistente com a literatura sobre a relação entre adição ao trabalho e saúde (Shimazu, Schaufeli \& Taris, 2010). Quanto maior o número de horas extras trabalhadas maior era a quantidade de relatos de estresse e problemas de saúde (Burke, 2000b; Sparks, Cooper, Fried \& Shirom, 1997; Spence \& Robbins, 1992; Tavares, Caetano \& Silva, 2007). De acordo com McMillan, O'Driscoll, Marsh e Brady (2001), este resultado pode estar relacionado ao sistema rígido de trabalho autoimposto pelo adicto e a não-permissão de recuperar seu esforço excessivo.

A satisfação com a vida geral relaciona-se negativamente com o TC. Esse resultado não surpreende, pois já vem sendo confirmado através de estudos realizados (Aziz \& Zickar, 2006; Bakker, Demeroti \& Burke, 2009; Echeburúa, 1999). Este resultado caracteriza o perfil do adicto ao trabalho, que percebe o trabalho como mais importante do que sua família, amigos e tempo livre. Estes costumam apresentar maior desequilibrio entre trabalho e vida pessoal (Aziz \& Zickar, 2006). Bakker, Demeroti e Burke (2009) hipotetizam se a adição não poderia ser uma consequência da insatisfação com a má relação afetiva, sendo esse comportamento uma estratégia de enfrentamento de fuga. O ser humano necessita lograr um nível de satisfação global com a vida, o qual, comumente, é alcançado dividindo-se em diversas atividades, como com a família, o trabalho, os relacionamentos afetivos e sociais, esporte e lazer de um modo geral. As carências em uma das dimensões importantes da vida poderão ser compensadas pela busca de satisfação em outras (Echeburúa, 1999).

O estudo apresenta algumas limitações que devem ser consideradas na análise de suas conclusões. A primeira é o delineamento transversal, o que impede conclusões em termos de causalidade. A segunda diz respeito ao efeito do trabalhador sadio, questão peculiar em estudos transversais em epidemiologia ocupacional que, muitas vezes, exclui o possível doente (Mc Michael, 1976). Essa é uma situação que pode subestimar o tamanho dos riscos identificados, porque os mais afetados não conseguem manter-se no emprego, afastando-se por licenças para tratamento de saúde. A terceira é que são utilizadas apenas medidas de autorelato, o que pode ocasionar algum viés devido à desejabilidade social que algumas questões abordam, no caso deste estudo, as questões relacionadas à sobrecarga de trabalho, pois, atualmente, conotações positivas de fluxo de trabalho árduo na nossa cultura reforçam padrões de comportamento aditivo. É socialmente aceitável trabalhar muito. De acordo com Killinger (1991), há um sentimento heroico em ter mais de um emprego para o sustento da família ou para proporcionar o padrão de vida desejável na atual sociedade de consumo. O trabalhador estabelece uma relação clara entre as horas trabalhadas e os benefícios obtidos, geralmente adota uma posição economicamente racional, ou seja, manter seu ritmo e dedicação ao trabalho (Del Líbano, Rodriguez, Llorens, Cifre \& Salanova, 2006; Moreno-Jiménez, GálvezHerrer, Garrosa-Hernández \& Rodríguez-Carvajal, 2005). Essa questão é preocupante, uma vez que todas as condutas aditivas estão controladas inicialmente por reforçadores positivos - o aspecto prazeroso da conduta em si -, mas terminam sendo controladas por reforçadores negativos - o alívio da tensão (Echeburúa,1999). A fuga e evasão de afeto negativo é o motivo preponderante nos comportamentos aditivos (Baker, Piper, McCarthy, Majeskie \& Fiore, 2004). Essas questões, semelhante ao que ocorre com as adições, dificultam o tratamento, uma vez que, não raras vezes, o nexo causal ocorre quando há um aprofundamento do quadro com sintomas e consequências bastante graves. Segundo AlonsoFernández (2008), o trabalhador não se reconhece como adicto, e quando o faz, sua conduta rígida o impede de dar seguimento às prescrições terapêuticas e adaptar-se a remodelação de seu estilo de vida. 


\section{Conclusão}

A adição ao trabalho começou a ser estudada há pouco tempo. O baixo número de investigações é uma das razões pelas quais ainda não se têm resultados muito conclusivos (Del Líbano, Llorens \& Salanova, 2007). Segundo Dudek (2008), ainda não se tem um conhecimento sólido sobre seu processo de desenvolvimento e suas consequências.

O presente estudo identificou um perfil de risco constituído por mulheres, profissionais com carga horária contratual e horas extras elevadas, com menos tempo de trabalho em geral e menos tempo na empresa atual, que se percebem menos saudáveis e que possuem menor satisfação com a vida em geral. $\mathrm{O}$ enfoque de risco assume que, quanto maior conhecimento sobre os eventos negativos, maior possibilidade de agir sobre eles com antecipação para evitá-los, mudando as condições facilitadoras para que um indivíduo ou grupo desenvolva a doença ou o dano (Álvarez, 2007).

$\mathrm{O}$ aumento da compreensão deste fenômeno psicossocial do mundo contemporâneo do trabalho implica na ampliação dos estudos sobre o tema. Del Líbano, Rodriguez, Llorens, Cifre e Salanova (2006) pontuam que ainda não são conhecidas com exatidão as razões da ocorrência da adição ao trabalho. Talvez, deva-se à pressão das próprias organizações de trabalho, as circunstâncias, particulares em que se encontram as pessoas, a fatores de personalidade ou pode ser um conjunto de todos esses fatores..

As investigações que são desenvolvidas atualmente tentam responder a essas questões devido à grande variedade de fatores implicados (Andreassen, Hetland, \& Le Pallesen, 2010). A partir de seu enfoque psicossocial, enfatiza-se a necessidade de estudos empíricos sobre fatores psicológicos buscando aprofundar características de personalidade, estratégias de enfrentamento, auto-eficácia, lócus de controle, dentre outras, assim como fatores sociais como aspectos econômicos, organizacionais e culturais. A adição ao trabalho deve ser melhor investigada devido a ampla variedade de fatores envolvido

$\mathrm{O}$ entendimento de seus resultados indicam a necessidade de ter-se como pano de fundo o atual momento da construção histórica do trabalho, se pensarmos na possibilidade da adição ao trabalho ser um fenômeno psicossocial, expressão de seu significado na pós-modernidade. O trabalho sofreu alterações ao longo da história adquirindo novos significados e valores. De uma atividade não digna de homens livres, foi utilizado como punição, tendo na modernidade, o entendimento de ser fonte crescimento e de realização pessoal. Oscilou entre o conceito de direito e cidadania ao de privilégio. Hoje, na pósmodernidade, representa inclusão social. $O$ sucesso alcançado por meio dele representa um estilo de vida diferenciado, considerado como uma característica de personalidade, pela qual os sujeitos passaram a ser avaliados em temos de inserção social (Appel-Silva \& Biehl, 2006). Seu foco é a eficácia e os resultados dele, não tendo importância a maneira como venham a ser alcançados e o quanto invade outros domínios da vida do trabalhador. A exigência da apropriação dos objetivos empresariais exige alta dedicação ao trabalho, apego emocional e envolvimento pessoal pelos sujeitos (Antunes, 2000). Para que as tarefas sejam cumpridas, há uma tendência de que os sujeitos realizem uma jornada de trabalho com um ritmo incessante e com muitas horas-extras, caso mal sucedido, corre o risco de demissão (Sennett, 2000). Os trabalhadores hoje se sentem responsáveis pelo próprio empregodesemprego, sucesso-fracasso e inclusão-exclusão social (Appel-Silva \& Biehl, 2006).

No caso da adição ao trabalho, o exame do contexto ampliado e do locus (nesse caso, as organizações) onde essa grave patologia psicossocial se manifesta pode lançar novas luzes sobre ela e auxiliar para a construção de um mundo do trabalho mais humanizado (Serva \& Ferreira, 2006).

\section{Referências}

Adorno, T. \& Horkheimer, M. (1985). Dialética do esclarecimento: fragmentos filosóficos. Rio de Janeiro: Jorge Zahar.

Alonso-Fernández, F. (2008)¿Por qué trabajamos?: el trabajo entre el estrés y la felicidad. Madrid: Díaz de Santos.

Álvarez, A. M. A. (2007). Fatores de risco que favorecem a recaída no alcoolismo. Jornal Brasileiro de Psiquiatria, 56(3),188-193.

Andreassen, C. S., Hetland,J., \& Le Pallesen, S. (2010). The Relationship Between 'Workaholism', Basic Needs Satisfaction at Work and Personality. European Journal of Personality, 24, 3-17.

Antunes, R. (2000). Os sentidos do trabalho: Ensaio sobre a afirmação $e$ a negação do trabalho. São Paulo: Boitempo.

Apple, M. W. (1995). Trabalho docente e textos: Economia política das relações de classe e gênero em educação. Porto Alegre: Artes Médicas.

Appel-Silva, M. \& Biehl, K. (2006). Trabalho na pósmodernidade: Crenças e concepções. Revista MalEstar e Subjetividade, VI(2), 518-534. 
Aziz, S. \& Zickar, M. J. (2006). A cluster analysis investigation of workaholism as a syndrome. Journal of Occupational Health Psychology, 11(1), 52-62.

Baker, T.B., Piper, M. E., McCarthy, D. E., Majeskie, M. R. \& Fiore, M. C. (2004). Addiction Motivation Reformulated: An Affective Processing Model of Negative Reinforcement. Psychological Review, 111(1), 33-51.

Bakker, A. B., Demerouti, E., \& Burke, R. (2009). Workaholism and relationship quality: a spillovercrossover perspective. Journal of Occupational Health Psychology, 14(1), 23-33.

Biernarcki, P. \& Waldorf, D. (1981). Snowball sampling-problems and techniques of chain referral sampling. Sociological Methods and Research, 10, 141-163.

Borrell, C. \& Artazcoz, L. (2008). Las desigualdades de género en salud: retos para el futuro. Revista Espanbola de Salud Pública, 82, 245-249.

Burke, R. J.(2000a) Workaholism and divorce. Psychology Repport, 86(1), 219-20.

Burke, R.J. (2000b). Workaholism in organizations: psychological and physical well-being consequences. Stress Medicine, 16, 11-6.

Burke, R. J. (2003). Workaholism: components among employees working full- and part-time. Psychology Repport, 93(1),84-86.

Burke, R. J. (2004). Workaholism, self-esteem, and motives for money. Psychology Repport, 86(1), 21920.

Burke, R. J. (2008). Work hours, work intensity, and work addiction. In: R. J., Burke \& C. L., Cooper (Orgs.), The long work hours culture: causes, consequences and choices (pp. 3-36). Bingley: Emerald Publishing.

Carlotto, M. \& Del Líbano (2010). Tradução, adaptação e exploração de propriedades psicométricas da Escala de Adição ao trabalho "Dutch Work Addiction Scale" (DUWAS). Contextos Clínicos, 3(2),141-150.

Cherrington, D.J. (1980). The work ethic: working values and values that work. New York: Amacom.

Codo, W. (1999). Educação: carinho e trabalho. Rio de Janeiro: Vozes.

Del Líbano, M., Renedo, M., Lorens, S. \& Salanova, M. (2005). ¿Existen relaciones significativas entre adicción al trabajo y satisfacción? Fòrum de Recerca.9, Disponível em Servei de Publicacions Universitat Jaume

http://.www.uji.es/bin/publ/

edicions/jfi9/psi/12.pdf .Acessado em: 02 jul 2008

Del Líbano, M., Llorens, S. \& Salanova, M. (2007). Adicción al trabajo: ¿un fenómeno positivo o negativo? Fòrum de Recerca,10. Disponível em Servei de Publicacions Universitat Jaume I. http://www.uji.es/bin/publ/edicions/jfi10/psi/1 3.pdf. Acessado em: 10 jul 2009.

Del Líbano, M., Llorens, S., Salanova, M. \& Schaufeli, W.B. (2010). Validity of a brief workaholism scale. Psychothema, 22, 143-150.

Del Líbano, M., Rodríguez, A. M., Llorens, S., Cifre, E. \& Salanova, M. (2006). Adicción al trabajo y Flow: una relación conflictiva. Riesgo Laboral, 14, 34-37.

Dudek, B. (2008). Workaholism - hazardous effect of excessive work involvement. Medycyna Pracy,59(3),247-254.

Echeburúa, E. (1999). ¿Adicciones... sin drogas? Las nuevas adicciones (juego, sexo, comida, compras, trabajo, internet). Bilbao. Desclée de Brouwer.

Hewlett S. A., \&, C. B. (2006). Extreme Jobs: The Dangerous Allure of the 70-Hour Workweek. Harvard Business Review. Disponível em http://hbr.org/2006/12/extreme-jobs/ar/1 Acessado em: 23 mai 2009

Killinger, B. (1991). Workaholic: the respectable addicts. New York: Simon \& Schuster.

Mc Michael, A. J. (1976). Standardized mortality ratios and the healthy worker effect: scratching beneath the surface. Journal Occupational Medical, $18,165-168$

McMillan, L. H. W., O'Driscoll, M.P., Marsh, N. V. \& Brady, E. C. (2001). Understanding workaholism: data synthesis, theoretical critique and future design strategies. International Journal of Stress Management. 8, 69-91.

Ministério da Saúde (1997). Conselho Nacional de Saúde. Diretrizes e normas para pesquisa envolvendo seres bumanos. Resolucão CNS 196/196. Brasília: Ministério da Saúde.

Miranda, D. \& Lobato, S. (2009). Processos de adoecimento ligados ao gênero: uma história de (des)valorização dos múltiplos papéis femininos. Disponível em: http://www.foxitsoftware.com. Acessado em: 08 Jun 2009.

Moreno-Jiménez, B., Gálvez-Herrer, M., GarrosaHernández, E. \& Rodríguez-Carvajal, R. (2005). $\mathrm{La}$ adiccion al trabajo. Psicología Conductual, 13(3),417-428.

Porter, G. (1996). Organizational impact of workaholism: suggestions for researching the negative outcomes of excessive work. Journal of Occupational Health Psychology, 1(1),70-84.

Porter, G. (2004). Work, work ethic, work excess. Journal of Organizational Change Management,17, 42439. 
Salanova, M., Llorens, S., Cifre, E. \& Martínez, I. M. (2006). Metodología RED-WONT. Perspectivas de intervención en riesgo psicosociales. Barcelona: Foment del Treball Nacional.

Salanova, M., Del Libano, M., Llorens, S., Schaufeli, W.B. \& Fidalgo, M. (2008). La adicción al trabajo. Nota Técnica de Prevención, 759, 22 ${ }^{\mathrm{a}}$ Serie. Instituto Nacional de Seguridad e Higiene en el trabajo, España.

Schaufeli, W. B., Taris, T. W. \& Bakker, A. (2006). Dr. Jekyll and Mr. Hide: on the differences between work engagement and workaholism. In: R. J., Burke (Org.), Research companion to working time and work addiction (pp. 193-217). Edward Elgar: Northampton, MA.

Schaufeli, W. B., Taris, T. W. \& Bakker, A. (2008). It takes two Tango: workaholism is working excessively and working compulsively. In: R. J., Burke \& C.L., Cooper, (Orgs.), The long work hours culture. Causes, consequences and choices (pp. 203-206). UK: Emerald.

Scott, K. S., Moore, K. S. \& Miceli, M. P. (1997). An exploration of the meaning and consequences of workaholism. Humans Relations. 50(3), 287-314.

Sennett, R. (2000). A corrosão do caráter. Rio de Janeiro: Record.

Serva, M. \& Ferreira, J. L. (2006). O. O fenômeno workabolic na gestão de empresas, RAP, 40(2),179200.

Shimazu, A. \& Schaufeli, W. B. (2009). Is workaholism good or bad for employee wellbeing? The distinctiveness of workaholism and work engagement among japanese employees. Industrial Health, 47, 495-502.

Shimazu , A. \&. Schaufeli, W. B. \& Taris, T.W. (2010). How does workaholism affect worker health and performance? The mediating role of coping. International Society of Behavioral Medicine, 17,154160.
Shiron, A. (1989). Burnout in work organizations. In: C. L., Cooper \& I. Robertson (Orgs.), International Review of Industrial and Organizational Psychology (pp. 25-48). New York: Wiley \& Sons.

Snir, R. \& Zohar, D. (2008). Workaholism as discretionary time investment at work: an experience-sampling study. Applied Psychology: An International Review, 57,109-127.

Sparks, K., Cooper, C., Fried, Y. \& Shirom, A. (1997).The effects of hours of work on health: A meta-analytic review. Journal of Organizational and Occupational Psychology, 51, 391-408.

Spence, J.T. \& Robbins, A. S. (1992). Workaholism: definition, measurement, and preliminary results. Journal of Personality Assessment, 58(1),160-78.

Taris, T.W. \& Schaufeli, W.B. (2007). Workaholism. In: W.B., Schaufeli \& A. B. Bakker (Orgs.), De psychologie van arbeid en gezondheid (pp. 359372). Houten: Bohn Stafleu vanLoghum.

Taris, T. W., Schaufeli, W. B. \& Verhoeven, L. C. (2005). Workaholism in the Netherlands: Measurement and Implications for Job Strain and Work-Nonwork Conflict. Applied Psychology An International Review,54(1), 37-60.

Tavares, S., Caetano, A. \& Silva, S. (2007). Não há bela sem senão: a identificação organizacional, os comportamentos de dedicação ao trabalho e o conflito trabalho-família. Psicologia, 21(1),133-149.

Thomas, W. H. N., Sorensen, K. L. \& Feldman, D. C. (2006). Dimensions, antecedents, and consequences of workaholism: a conceptual integration and extension. Journal of Organizational Behavior, 28(1), 111-136.

Tucker, P. \& Rutherford, C. (2005). Moderators of the relationship between long work hours and health. Journal of Occupational Health Psychology, 10(4),465-76.

Recebido em abril de 2010 Reformulado em outubro de 2010 Aprovado em dezembro de 2010

Sobre a autora:

Mary Sandra Carlotto é Psicóloga, Mestre em Saúde Coletiva (Universidade Luterana do Brasil/ULBRA-Canoas), Doutora em Psicologia Social (Universidade de Santiago de Compostela-USC/Espanha) e Professora da Pontifícia Universidade Católica do Rio Grande do Sul. Bolsista produtividade do CNPq. 